\title{
Robust and cost-effective system for measuring and logging of data on soil water content and soil temperature profile
}

\author{
Mitja Ferlan ${ }^{1,2^{*}}$, Primoz Simončič ${ }^{2}$ \\ ${ }^{1}$ University of Ljubljana, Biotechnical Faculty, Ljubljana, Slovenia; ${ }^{*}$ Corresponding Author: mitja.ferlan@gozdis.si \\ ${ }^{2}$ Slovenian Forestry Institute, Ljubljana, Slovenia
}

Received 13 June 2012; revised 22 July 2012; accepted 10 August 2012

\begin{abstract}
The paper describes the system for measuring and logging of data on soil water content and soil temperature profile. The system was tested in a field and shows great potential for performing continuous measurements. It has several benefits including ease of manufacture, low cost, reliable performance and the ability to download the data without specialized software.
\end{abstract}

Keywords: Data-Logger; Soil Temperature; Soil Water Content; Microcontrollers; Sensors

\section{INTRODUCTION}

Continuous and automated systems for measuring and data-logging are needed nowadays in almost every natural environmental research project. Each natural environment or ecosystem is composed of different segments. For all terrestrial ecosystems, the common segment is soils, where numerous processes take place. One of the most important is soil respiration, which is also, after photosynthetic assimilation, the second largest flux of carbon and one of the key determinants of net-ecosystem carbon exchange [1]. To measure soil respiration, the researchers have used different techniques. Simple but time consuming technique is using close dynamic or open dynamic chambers [2] and portable infrared gas analyzer with an appropriate data logger and system for pumping air. The weakness of portable system for measuring soil respiration is non-continuous measurements. One of such portable system for measuring soil respiretion with close dynamic chamber is LI-6400 (LI-COR Biosciences Inc., Lincoln, NE). More complicate and more expensive but less time consuming is the use of automated systems for measuring soil respiration (e.g.: LI-8100, LI-COR Biosciences Inc., Lincoln, NE). Main feature of these systems is continuous measurements of soil respiration. Another expensive way to measure soil respiration is the profile method, but it is not widely used in comparison with the previously mentioned methods [3]. In natural environments, high spatial variability, especially in soils, is not rare and therefore several repetitions of soil respiration measurement are needed. The main drivers of soil respiration are soil temperature (Ts) and soil water content (SWC). Continuous measurements of these two parameters can be relatively easily performed. In the case of using portable systems for measuring soil respiration, we are limited to sensing temporal variability but in the case of using automated systems we are limited to sensing spatial variability. Both limitations can be minimized with additional measurements of Ts and SWC using apropriate model to gap-fill the data [4]. Spatial and temporal variability of these two parameters could be observed with an appropriate number of Ts and SWC profiles. Several profiles mean that many connections with cables must be made, or alternatively, expensive installation of wireless sensors could be used. Due to these needs, we have developed, constructed and tested a robust and cost-effective system for measuring and data logging Ts and SWC, which is presented in this paper.

\section{SYSTEM DESCRIPTION}

The system for measuring and data logging soil water content and soil temperature profiles is microcontrollerbased with sensors and peripheral components connected to it. All selected electronic components have low power consumption and allow battery-powered operation. Cost estimation and electronic parts of the system are listed in Table 1.

\subsection{Unit for Measuring Soil Water Content}

Two frequency domain sensors EC-5 (Decagon Devices Inc., Pullman, WA) for measuring soil water content were used. For correct supply voltage of $2.5 \mathrm{~V}$ for 
Table 1. Part list of the system with cost estimation.

\begin{tabular}{cccc}
\hline Description & Part number & Quantity & Cost $(€)$ \\
\hline Conectors & Male pins & 5 & 0.50 \\
Voltage regulator & REG1117 & 1 & 0.40 \\
Memory & AT24C512 & 1 & 3.40 \\
Microcontroller & ATMEGA16 & 1 & 4.40 \\
Capacitators & & 8 & 0.10 \\
CRYSTAL & Clock (32.768 kHz) & 1 & 0.70 \\
Chokes & & 2 & 0.20 \\
Resistors & & 4 & 0.10 \\
Diode & 1N4148 & 1 & 0.10 \\
Batery pack & $4 x A A$ alkaline & 1 & 5.20 \\
Waterproof enclosure & & 1 & 9.50 \\
Circuit boards & & - & 7.40 \\
Cables & DS18B20 & 7 & 24.50 \\
Mount material & & - & 2.00 \\
Soil water content sensor & EC-5 & 2 & 130.00 \\
Temperature sensors & & & \\
Serial converter & MAX232 & 1.50 \\
\hline
\end{tabular}

the sensors, regulator LD1117 (SGS-THOMSON Microelectronics) was used. Sensor output ranges from 250 to $1000 \mathrm{mV}$ at a $2.5 \mathrm{~V}$ supply voltage and supposed to be proportional to volumetric soil water content.

The central unit has a built-in 10-bit analog to digital converter (ADC converter). Therefore only a $100 \mathrm{mH}$ choke and a $100 \mathrm{nF}$ capacitor must be applied to perform an accurate voltage reading. Due to this feature, there is no need to construct a peripheral analog to digital converter and fewer components are needed; consequently the price is lower. The system described in this paper has two single ended channels for measuring voltages between 0 and $2500 \mathrm{mV}$. Since any other sensors with output signal from 0 to $2500 \mathrm{mV}$ can be used with the presented system, only an accuracy test of voltage readings with our system was performed. For this purpose we used a laboratory voltage generator (Digimaster,

DF1730SB5A) generating voltage in range from 0 to $2500 \mathrm{mV}$. Voltage was measured with our system and simultaneously with a laboratory Digital Mutilmeter (M3890D-USB, Metex Instruments, Seoul, Korea) connected to a PC for logging. Values were logged every second. Linear regression between our system and Digital Mutilmeter was done $\left(\mathrm{n}=1548, \mathrm{R}^{2}=0.998\right.$, slope $=$ 1.002 , intercept $=-0.188$ ). From these results we can see that our system underestimates voltage by aproximately
$0.2 \mathrm{mV}$; for equipment that can measure voltage from 0 to $2500 \mathrm{mV}$ this is neglible. From simultaneously measurements accuracy of system voltage measurements was calculated and it is $+-0.22 \%$ for testing range.

\subsection{Unit for Measuring Temperature}

For measuring temperature, most data loggers use differential or single-ended voltage measurements and different types of sensors. Most frequently used are thermocouples or thermo-sensitive resistors with negative or positive temperature coefficients. There are also several types of integrated circuits which can measure temperature and convert data to a digital signal. When several sensors must be used and are connected to the same measurement and data-logging system, usually at least two cables per sensor must be used and several voltage or digital input channels are needed. The system described in this paper uses factory calibrated temperature sensors DS18B20 (Maxim Integrated Products, Sunnyvale, CA) with $\pm 0.5^{\circ} \mathrm{C}$ accuracy in the range between $-10^{\circ} \mathrm{C}$ and $+85^{\circ} \mathrm{C}$. The sensors use 1-wire communication protocol (1-Wire is a registered trademark of Maxim Integrated Products, Inc). The most important feature of this sensor is that several sensors (up to 100) can be connected in series on the same cable. Each sensor has a unique serial number and therefore the central unit can communicate with a certain sensor and obtain its temperature reading. The cable for short distances can have one wire for data transfer and power supply and another for ground; for long distances, one more wire is needed for power-supply. In any case, the 1-wire protocol requires only one pin for the microcontroller to communicate with several sensors. Reducing the number of cables reduces the risk of damage to cables (by rodents etc.) and the risk of loss of data. For measuring a temperature profile in soil, the sensors can be installed on a specially designed printed circuit with length of $60 \mathrm{~cm}$, according to World Meteorological Organization (WMO) standards to sense temperature at elevations of $-50 \mathrm{~cm},-30 \mathrm{~cm}$, $-20 \mathrm{~cm},-10 \mathrm{~cm},-5 \mathrm{~cm},-2 \mathrm{~cm}$ and $5 \mathrm{~cm}$ with respect to the soil surface. The circuit is connected to the datalogger via a three-wire cable and placed in a plastic tube with diameter $13 \mathrm{~mm}$ and length $65 \mathrm{~cm}$, insulated with foam. This tube for measurement of soil temperature profile is easier to install in soil and reduces the potential damage to temperature sensors. For the placement of the tube for measuring soil temperature profile in rocky soil, a drill with appropriate drilling machine could be used, and in other types of soil an appropriate hand auger could be used. For sensors placed $5 \mathrm{~cm}$ above the ground, a special home-made radiation shield was used. For measuring temperature with presented system only 1wire sensors DS18B20 could be use, or any other tem- 
perature sensors with output ranged from 0 to $2500 \mathrm{mV}$ can be connected to single ended channels. Despite manufacturer guarantee that sensors are calibrated, we performed a test with seven temperature sensors placed in a measurement stick and classical meteorological thermometer (mercury thermometer). As a testing media, water with ice was used and appropriate hand mixing was performed. Temperature ranged from $4^{\circ} \mathrm{C}$ to $17^{\circ} \mathrm{C}$ and values were logged or manually read every $30 \mathrm{~min}$ utes. For each temperature sensor, linear regression with mercury thermometer measurements $(n=12)$ was made. From all parameters of linear regression $(\mathrm{N}=7)$, we calculated means and standard deviations $\left(\mathrm{R}^{2}=0.997+\right.$ -0.001 , slope $=0.975+-0.023$, intercept $=0.124+$ $-0.377)$. With these temperature sensors, the temperature is slightly underestimated compared with the mercury thermometer. From simultaneously measurements accuracy of system temperature measurements was calculated and it is $\pm 1.1 \%$ for testing range.

\subsection{Unit for Data Logging}

The Philips Inter-IC communications (I2C) protocol (Philips Semiconductors, The Netherlands) is commonly used for interfacing peripheral devices and microcontrollers. We used this type of communication for datalogging of nine measured parameters and time stamp on an AT24C512 (Atmel Corporation, San Jose, CA) memory module. Measurement frequency in range from 5 to 3600 s can be set and system informs us for how many days the data-logger can store data (For example: measuring frequency set to 30 minutes, the data-logger can store data for 59 days). If we do not download the data to an external device (notebook, handheld computer...) and the memory module is full, the microcontroller stops measuring and goes into power-down mode.

\subsection{Unit for Communication with Other Devices}

The user can communicate with the circuit using terminal software on a notebook or handheld computer. Communication is available via serial interface (baud rate: 56,000, Data bits: 8 , Parity: none, Stop bits: 1, Handshaking: none). To establish serial communication with computer also serial to USB converter could be used. When connection with the computer is established the microcontroller program starts a user-interface routine and displays in the terminal program a notice with the instructions on how to set up time and date, the location of data-logger or download the data. One of the advantages of the presented system for measuring and data-logging is that you do not need to install any special software on your computer. Hyper Terminal (Windows) or any other free software for reading data from serial ports can be used.

\subsection{Unit for Constant Power Supply with Battery Pack}

The microcontroller circuit is powered by a battery pack, consisting of four AA-size, $1.5 \mathrm{~V}$ alkaline batteries. Since the microcontroller and other devices (except sensors EC-5 for which a voltage regulator is used) can operate at a voltage between 4.5 and $5.5 \mathrm{~V}$, only a diode was used in the supply line to avoid short-circuits and to reduce the voltage from the battery pack. The current drawn is approximately $15 \mu \mathrm{A}$ in power save mode and $22 \mathrm{~mA}$ during measurement. Each measurement lasts less than 2 seconds and if the measurement frequency is set to 30 minutes, using standard AA alkaline batteries, with a capacity of approximately $2500 \mathrm{mAh}$, we can expect a battery life of approximately 5.5 years.

\subsection{Central Unit with Real-Time Clock and Running Program}

For the central unit a microcontroller (ATmega16, Atmel Corporation, San Jose, CA) was used. The ATmega16 is a low-power CMOS 8-bit microcontroller based on the AVR enhanced RISC architecture. The ATmega16 offers several functions that allow the construction of the system with minimum peripheral components, because of which it is cost-effective and robust. To apply a real-time clock, the crystal oscillator with $32.768 \mathrm{kHz}$ frequency must be connected to the microcontroller.

The software for the microcontroller was written in a BASIC-like language (BASCOM-AVR, MCS Electronics, Holland) for Microsoft Windows XP. The program was compiled in microcontroller assembly language and uploaded to the microcontroller using a programmer (PROGGY AVR, AX Elektronika, Slovenia) connected to a desktop computer via AVR Studio 4 software (Atmel Corporation, San Jose, CA).

\subsection{System Fabrication and Housing}

The system with all its components was built by the authors, who have also designed the electrical schematic of the circuit board (Figure 1). The schematic was transferred to the circuit board, which was drilled out on a small CNC machine in the laboratory for electronic systems at the Slovenian Forestry Institute.

Electronic components and connectors were soldered onto each circuit board and sensor cables were prepared. The tubes for temperature profile measurements were prepared according to World Meteorological Organization standards. For the fabrication and testing of one system approximately 4 hours are required.

The system has a waterproof housing. Three cables for 


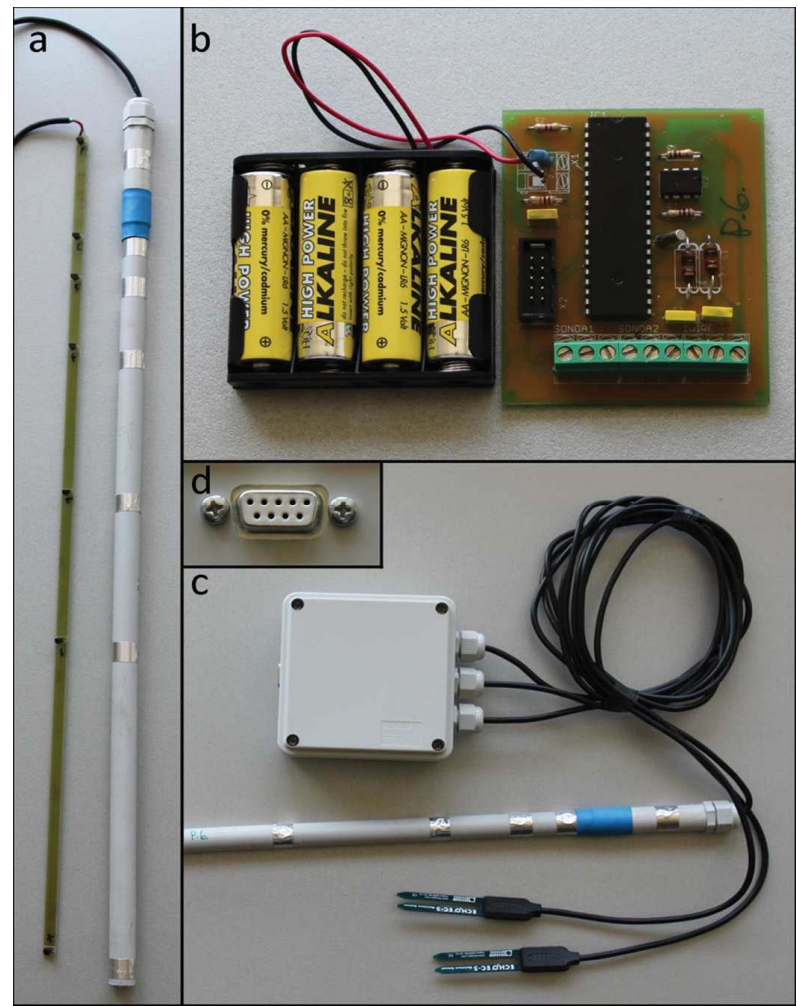

Figure 1. (a) Circuit board with sensors DS18B20 and finished measurement stick; (b) Microcontroller circuit board with battery pack; (c) Waterproofed housing with system for measuring and logging soil temperature profile and soil water content; (d) Female DB9 connector for serial communication with the system built outside.

sensors enter the housing through waterproof cable glands. The other side of the box houses a female DB9 connector. To protect the female DB9 connector from humidity and water, a male DB9 connector was made and used as a waterproof cover. The system housing allows a very convenient field installation and data downloading. The schematic is shown on Figure 2, circuit board (CNC or CAM format) and program (BASIC text or compiled) are available by contacting the authors.

\section{FIELD MEASUREMENTS AND TESTING OF THE SYSTEM}

The system was tested in an abandoned karstic pasture in SW Slovenia, Europe [5]. Soil respiration measurements were performed under forest and in gaps between forests [6]. Supporting measurements were performed by establish six soil water content and temperature profiles. According to the manual for soil water content sensor EC-5, measured $\mathrm{mV}$ were transformed to $\%$ using the suggested equation for mineral soils with accuracy of + $-5 \%$. Measurements started in February 2010. Precipitation measurements, measurements of soil water content using two time domain reflectometers (CS616, Campbell
Scientific, Logan, UT USA) inserted horizontally at 10 $\mathrm{cm}$ and soil temperature at the same level using thermocouples (TCAV, Campbell Scientific, Logan, UT USA) were made in a nearby meteorological station.

Because of rocky soils, tubes for measuring temperature profiles were inserted in holes that were drilled into the soil with a $14 \mathrm{~mm}$ diameter drill. The sensors for soil water content were inserted horizontally into a dug soil pit (10 and $30 \mathrm{~cm}$ depth). One of instalation was done near meteorological station soil profile, to compare new system with typical device.

A notebook was used to download data during periodic visits. The data were stored and later imported into Stata 7.0 software, where they were checked and appended to the database. In the first few months, small lead-acid rechargeable batteries $(6 \mathrm{~V}, 1.2 \mathrm{Ah})$ were used. The downside of these batteries is high self-discharge during higher temperatures - because of this, we have some missing data in April 2010. After replacing the lead-acid batteries with battery packs, consisting of four AA $1.5 \mathrm{~V}$ alkaline batteries, we did not have any problems with data loss.

Soil temperature and soil water content data to compare new device with typical device were measured at 10 $\mathrm{cm}$ by near meteorological station and dataset of year 2010 was used for comparison. Linear regressions were performed for temperature and for soil water content measurements (Ts: $\mathrm{R}^{2}=0.9976$, slope $=0.9912$, intercept $=0.2744$, SWC: $\mathrm{R}^{2}=0.9614$, slope $=0.8315$, intercept $=0.0338$ ). From these results we can see that our system systematicly underestimates temperature for aproximately $0.27^{\circ} \mathrm{C}$ and overestimate soil water content, especialy at higher values of this parameter (Figure 3). Differences in soil water content measurements are aslo present because construction of EC-5 sensor differ from CS616 sensor.

Example data for a 14-day period between 16th May and 29th May is shown in Figure 4.

Concerning the temperature conditions, higher temporal variability in the plot between forests was observed. The temperature $5 \mathrm{~cm}$ above the ground can be almost $20^{\circ} \mathrm{C}$ higher in the plot between forests than in the forest. On average, mean soil temperature in the forest is $13.5^{\circ} \mathrm{C}$ and the temperature between forests is $1.8^{\circ} \mathrm{C}$ higher. During rain on 21st May, approximately the same temperature was measured in both plots. As far as content of soil water is concerned, higher values were measured in the forest. On average, mean soil water content in the forest period is $8.4 \%$ higher (28.7\%) in comparison with the plot between forests $(20.3 \%)$ during the displayed period. Also the dynamics of soil water content between and after rain events are different between plots. This is especially evident during the vegetation period and differences could be observed between plots. 


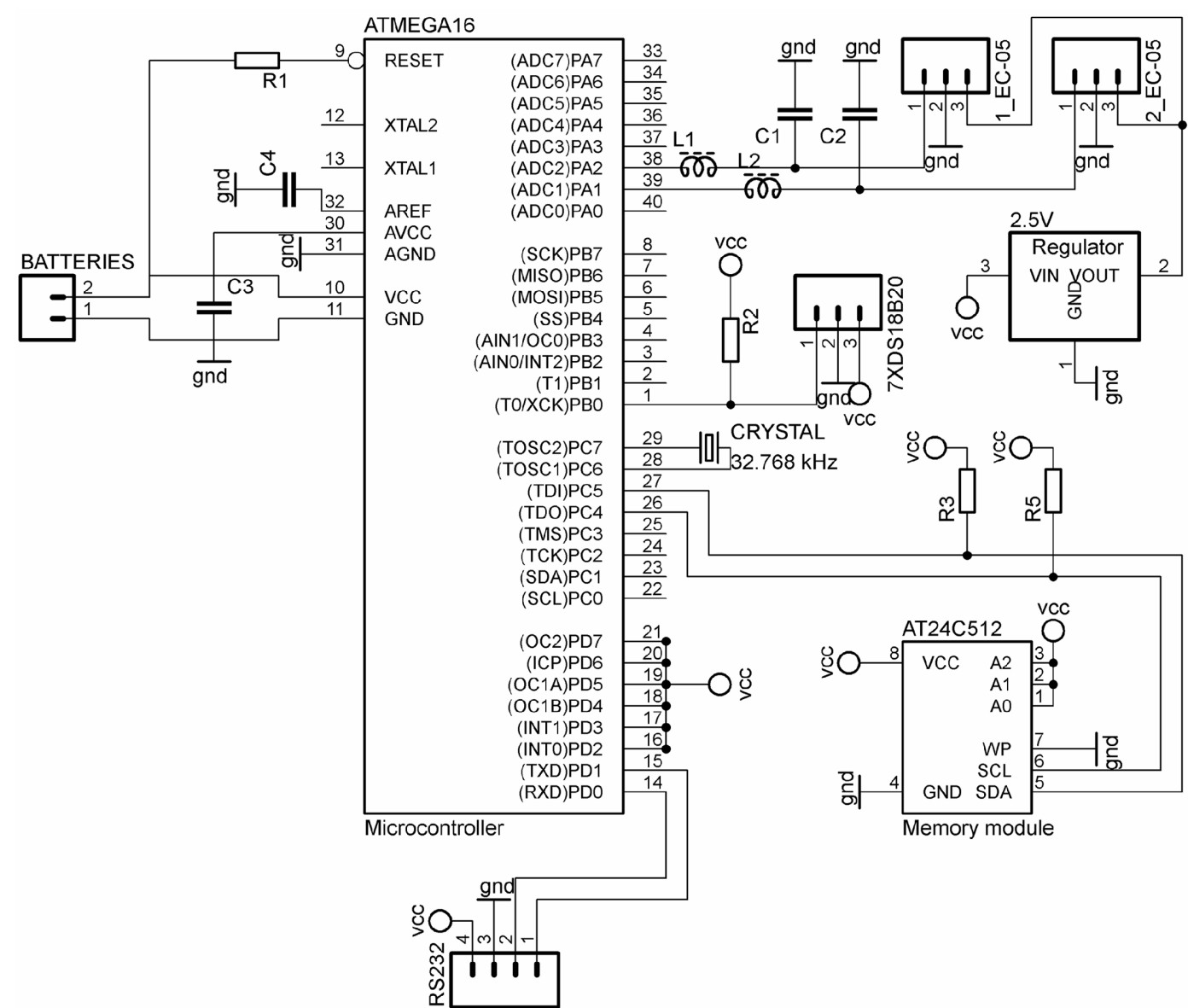

Figure 2. Electrical schematic of circuit board.

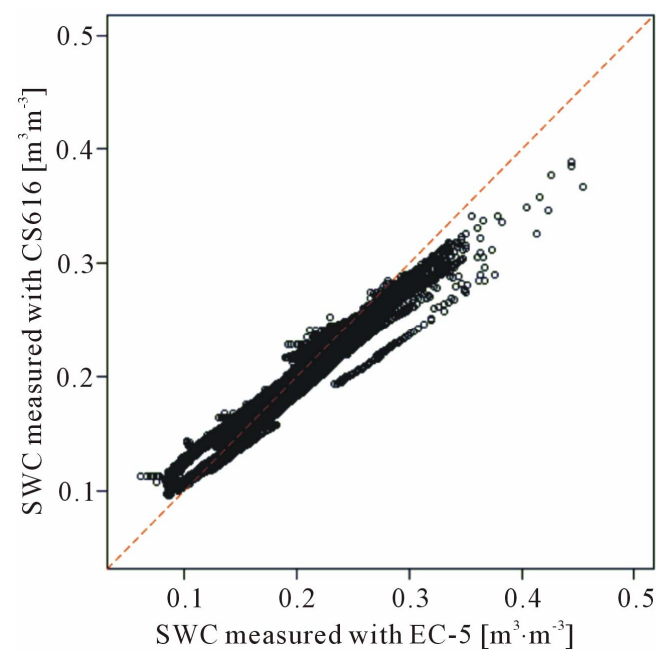

(a)

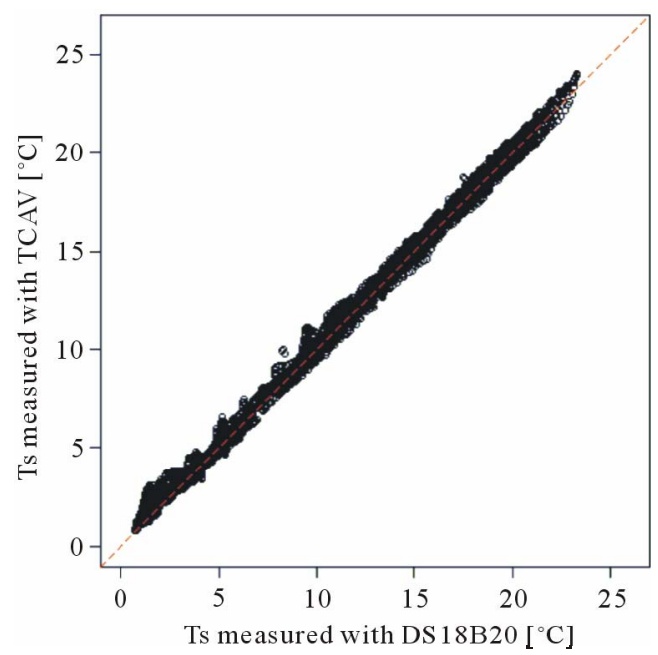

(b)

Figure 3. Comparison of new system with typical device. (a) Soil water content measuremetns with EC-5 and CS616; (b) Soil temperature measurements with DS18B20 and TCAV.

After a rain event on 27th May, soil water content in forest at $30 \mathrm{~cm}$ depth (brown line) did not reach as high level as observed on the plot between forests. Intensive leaf emergence in the beginning of the vegetation period was observed after rain on 21st May and the lack of soil moisture at $30 \mathrm{~cm}$ in the forest was due to interception. Detailed analyses could be drawn from the shown data, but this would exceed the scope of this study. 

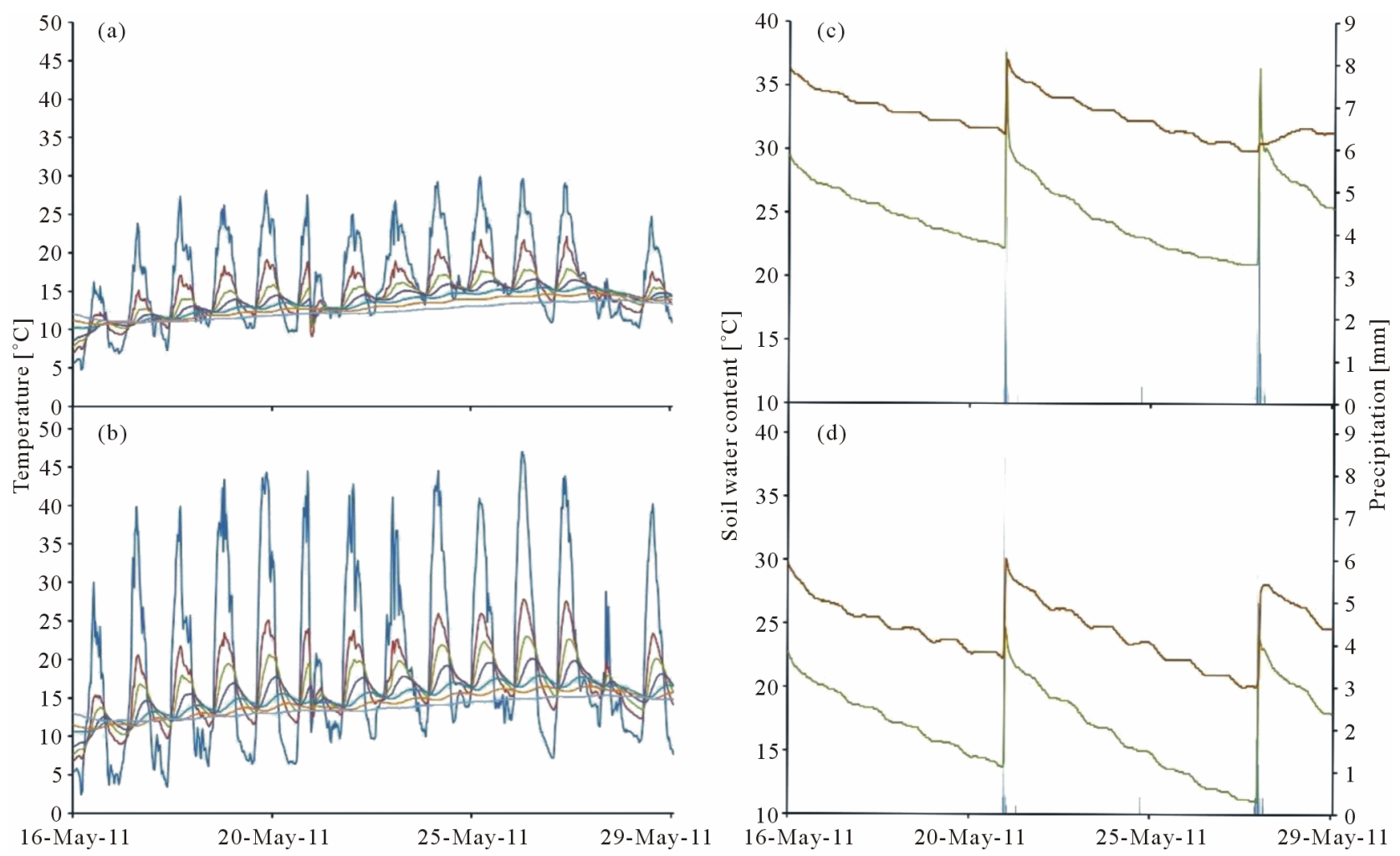

Figure 4. Example data of soil water content and soil temperature profile for 14-day period between 16th May and 29th May. (a) Soil temperature profile for plot in forest fragment (air temperature at $5 \mathrm{~cm}$ above ground (dark blue line), soil temperature at $2 \mathrm{~cm}$ (red line), $5 \mathrm{~cm}$ (green line), $10 \mathrm{~cm}$ (purple line), $20 \mathrm{~cm}$ (blue line), $30 \mathrm{~cm}$ (orange line), $50 \mathrm{~cm}$ (grey line)); (b) Soil temperature profile for plot between forest fragments (air temperature at $5 \mathrm{~cm}$ above ground (dark blue line), soil temperature at $2 \mathrm{~cm}$ (red line), $5 \mathrm{~cm}$ (green line), $10 \mathrm{~cm}$ (purple line), $20 \mathrm{~cm}$ (blue line), $30 \mathrm{~cm}$ (orange line), $50 \mathrm{~cm}$ (grey line)); (c) Soil water content for plot in forest fragment (soil water content at $10 \mathrm{~cm}$ (green line), soil water content at $30 \mathrm{~cm}$ (brown line)); (d) Soil water content for plot between forest fragments (soil water content at $10 \mathrm{~cm}$ (green line), soil water content at $30 \mathrm{~cm}$ (brown line)).

\section{CONCLUSION}

The example presents only one aspect of practical use of the measurements, collected with our system. If we would install our system into a systematic grid for example, we would have a presentation of spatial and temporal variability in the natural ecosystem. This feature can be very useful in inhomogeneous ecosystems such as abandoned extensive pasture. The information collected using the microcontroller-based system for measuring and logging of soil water content and temperature profile has helped with modeling soil respiration between periodic measurements on the abandoned extensive pasture. Furthermore, with additional analyses of soil in the laboratory, the data from soil temperature profiles will be used to calculate soil heat flux, which is one of the components of the energy balance of the ecosystem.

\section{REFERENCES}

[1] Raich, J.W. and Schlesinger, W.H. (1992) The global carbon-dioxide flux in soil respiration and its relationship to vegetation and climate. Tellus Series B-Chemical and Physical Meteorology, 44, 81-99. doi:10.1034/j.1600-0889.1992.t01-1-00001.x

[2] Hutchinson, G.L. and Livingston, G.P. (2001) Vents and seals in non-steady-state chambers used for measuring gas exchange between soil and the atmosphere. European Journal of Soil Science, 52, 675-682. doi:10.1046/j.1365-2389.2001.00415.x

[3] Nagy, Z., Pinter, K., Pavelka, M., Darenova, E. and Balogh, J. (2011) Carbon fluxes of surfaces vs ecosystems: Advantages of measuring eddy covariance and soil respiration simultaneously in dry grassland ecosystems. Biogeosciences, 8, 2523-2534. doi:10.5194/bg-8-2523-2011

[4] Lloyd, J. and Taylor, J.A. (1994) On the temperature dependence of soil respiration. Functional Ecology, 8, 315323. doi: $10.2307 / 2389824$

[5] Ferlan, M., Alberti, G., Eler, K., Batič, F., Peressotti, A., Miglietta, F., Zaldei, A., Simončič, P. and Vodnik, D. (2011) Comparing carbon fluxes between different stages of secondary succession of a karst grassland. Agriculture, Ecosystems and Environment, 140, 199-207. doi:10.1016/j.agee.2010.12.003

[6] Plestenjak, G., Eler, K., Vodnik, D., Ferlan, M., Čater, M., Kanduč, T., Simončič, P. and Ogrinc, N. (2012) Sources of soil $\mathrm{CO}_{2}$ in calcareous grassland with woody plant encroachment. Journal of Soils and Sediments, in Press. doi:10.1007/s11368-012-0564-3 